\title{
Holmboe wave fields in simulation and experiment
}

\author{
J. R. CARPENTER $\dagger$, E. W. TEDFORD, \\ M. RAHMANI AND G. A. LAWRENCE \\ Department of Civil Engineering, University of British Columbia, Vancouver, BC V6T 1Z4, Canada
}

(Received 21 March 2009; revised 31 October 2009; accepted 2 November 2009)

The basic wave field resulting from Holmboe's instability is studied both numerically and experimentally. Comparisons between the direct numerical simulations (DNS) and laboratory experiments result in Holmboe waves that are similar in their appearance and phase speed. However, different boundary conditions result in mean flows that display gradual variations either temporally (in the simulations) or spatially (in the experiments). These differences are found to affect the evolution of the dominant wavenumber and amplitude of the wave field. The simulations exhibit a nonlinear 'wave coarsening' effect, whereby the energy is shifted to lower wavenumbers in discrete merging events. This process is typically found to result from either ejections of mixed fluid away from the density interface or vortex pairing. In the experiments, energy is transferred to lower wavenumbers by the 'stretching' of the wave field by a gradually varying mean velocity. This stretching results in a reduction of wave amplitude compared with the DNS.

\section{Introduction}

Geophysical flows often exhibit stratified shear layers in which the region of density variation is thinner than the thickness of the shear layer (e.g. Wesson \& Gregg 1994; Yoshida et al. 1998; Sharples, Coates \& Sherwood 2003; Tedford et al. $2009 a$ ). In these circumstances, when the stratification is sufficiently strong (measured by an appropriate Richardson number), Holmboe's instability develops (Holmboe 1962). At finite amplitude the instability is characterized by cusp-like internal waves (referred to herein as Holmboe waves) that propagate with respect to the mean flow. Accurate modelling of these instabilities is important for the correct parameterization of momentum and mass transfers occurring in flows of this nature.

Previous studies on the nonlinear behaviour of Holmboe waves have adopted one of two methods: either an experimental approach in which the instability is studied under specified laboratory settings (Pouliquen, Chomaz \& Huerre 1994; Zhu \& Lawrence 2001; Hogg \& Ivey 2003) or a numerical approach that allows for a detailed description of the flow in an idealized stratified mixing layer (Smyth, Klaassen \& Peltier 1988; Smyth \& Winters 2003; Smyth 2006; Smyth, Carpenter \& Lawrence 2007). It is difficult to make a meaningful comparison of laboratory and numerical results for a number of reasons. In the caseof laboratory experiments, Holmboe waves

$\dagger$ Present address: EAWAG, Swiss Federal Institute of Aquatic Science and Technology, Seestrasse 79, Kastanienbaum, Switzerland. Email address for correspondence: jeffcarp@gmail.com 
often arise as a local feature of a larger-scale flow, such as an exchange flow between two basins of different density (Pawlak \& Armi 1996; Zhu \& Lawrence 2001; Hogg \& Ivey 2003), or an arrested salt wedge flow (Sargent \& Jirka 1987; Yonemitsu et al. 1996). Often in these experiments the mean flow varies appreciably over length scales that are comparable to the wavelength of the waves. For this reason, it can be difficult to isolate the dynamics of the waves from that of the mean flow.

The use of numerical simulations has been advantageous in this regard and comprises a great majority of the literature on the nonlinear dynamics of Holmboe waves. The first verification of two oppositely propagating cusp-like waves of equal amplitude, predicted by the theory of Holmboe (1962), was made through the numerical simulations of Smyth et al. (1988). Since then, increases in computational resources have led to fully three-dimensional direct numerical simulations (DNS) of Holmboe waves that resolve the smallest scales of variability. These simulations have been used to understand turbulence and mixing characteristics (Smyth \& Winters 2003; Carpenter, Lawrence \& Smyth 2007; Smyth et al. 2007), as well as the growth of secondary circulations and the transition to turbulence (Smyth 2006). However, with the exception of Alexakis (2009), only a single wavelength of the primary instability has been reported in the literature. Furthermore, no attempt has been made to compare the results of numerical simulations with laboratory experiments.

In the current paper, we undertake a combined numerical and experimental study of Holmboe waves. The experiments, originally described by Tedford, Pieters \& Lawrence (2009b), consist of an exchange flow through a relatively long channel with a rectangular cross-section. The experimental design allows for a detailed study of the Holmboe wave field within a steady mean flow that exhibits gradual spatial variation relative to the wave properties. The DNS of the present study were designed to correspond as closely as possible to the conditions present in the experiments to effect a meaningful comparison between the two methods. To our knowledge, this is the first study to compare experimental and numerical results, as well as the first to perform three-dimensional DNS for multiple wavelengths of the Holmboe instability. We focus on comparing basic descriptors of the wave fields, such as phase speed, wavenumber and wave amplitude, in order to gain a fuller understanding of the processes affecting the nonlinear behaviour of the waves.

The paper is organized as follows. Section 2 gives a background on the stability of stratified shear flows. This is followed by a description of the numerical simulations and laboratory experiments in $\S 3$. We then discuss comparisons between the simulations and experiments in terms of the basic wave structure ( $\$ 4)$, phase speed $(\S 5)$, wave spectral evolution $(\S 6)$ and wave amplitude and growth $(\S 7)$. Conclusions are stated in the final section.

\section{Linear stability of stratified shear layers}

In both experiment and simulation, the mean flow exhibits the characteristics of a classic stratified shear layer. The velocity profile undergoes a total change of $\Delta U$, over a length scale $h$, that is centred with respect to the density interface. Similarly, the density profile changes by $\Delta \rho$ between the two layers, over a scale of $\delta$. This suggests using an idealized model of the horizontal velocity and density profiles that 

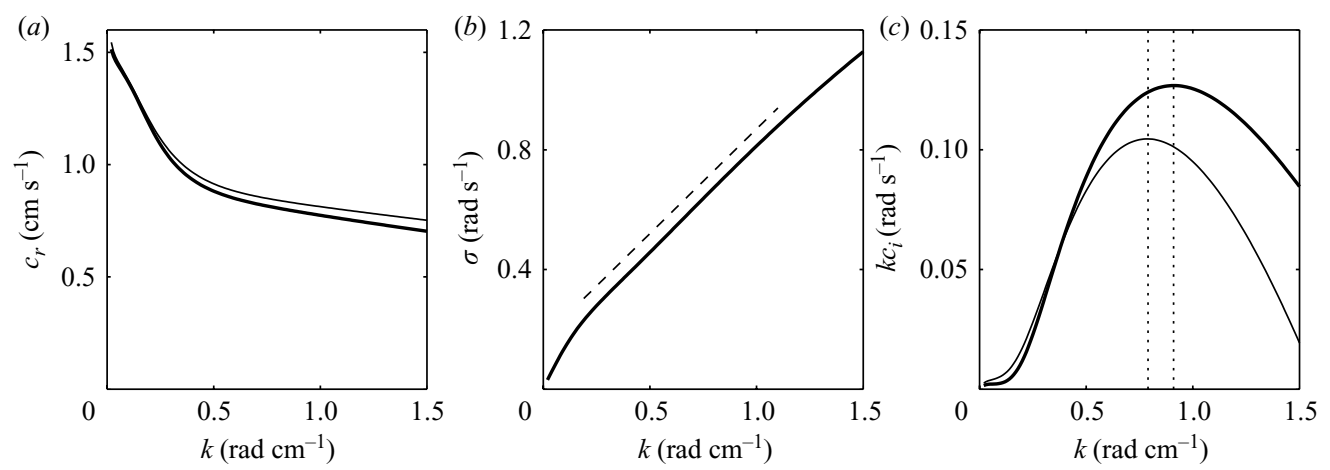

Figure 1. Plots of $(a)$ phase speed $c_{r},(b)$ frequency $\sigma$ and $(c)$ growth rate $k c_{i}$, of the profiles in (2.1). Conditions in the laboratory experiment are shown as the thick lines, with the three-dimensional simulation at $\operatorname{Pr}=25$ and $R=5$ as the thin lines and the dashed line in (b) indicating $\sigma \propto k$. No noticeable difference between the simulation and the experiment can be seen in $(b)$. The locations of the wavenumber of maximum growth are marked with a vertical dotted line in $(c)$.

is given by

$$
U(z)=\frac{\Delta U}{2} \tanh \left[\frac{2\left(z-z_{0}\right)}{h}\right] \text { and } \bar{\rho}(z)=\rho_{0}-\frac{\Delta \rho}{2} \tanh \left[\frac{2\left(z-z_{0}\right)}{\delta}\right],
$$

respectively. The density profile $\bar{\rho}(z)$ is measured relative to a reference density $\rho_{0}$, with $z$ the vertical coordinate. A necessary condition for the growth of Holmboe's instability is that the thickness ratio $R \equiv h / \delta>2$ (Alexakis 2005, 2007).

In addition to $R$, we can define three more important dimensionless parameters as follows:

$$
R e \equiv \frac{\Delta U h}{\nu}, \quad J \equiv \frac{g^{\prime} h}{(\Delta U)^{2}} \quad \text { and } \quad P r \equiv \frac{v}{\kappa},
$$

where $g^{\prime}=\Delta \rho g / \rho_{0}$ is the reduced gravitational acceleration; $v$ is the kinematic viscosity; and $\kappa$ the diffusivity of the stratifying agent. These are the Reynolds, bulk Richardson and Prandtl numbers, respectively.

Linear stability analysis of the profiles in (2.1) has been performed in numerous studies (e.g. Hazel 1972; Smyth et al. 1988; Haigh 1995). For the flows considered here, the effects of viscosity and mass diffusion have been included. The resulting equation is a sixth-order eigenvalue problem originally described by Koppel (1964). Like the better-known Taylor-Goldstein equation, Koppel's equation gives predictions of the complex phase speed $c=c_{r}+\mathrm{i} c_{i}$, and vertical mode shape, as a function of wavenumber $k$. Results of the stability analysis are shown in figure 1, which includes the dispersion relation in terms of phase speed $c_{r}(k)$ and frequency $\sigma(k)$, as well as the temporal growth rate $k c_{i}$. This is done for the idealized profiles (2.1) using $R e=630, J=0.30, \operatorname{Pr}=700$ and $R=8$, matching the dimensionless parameters in the laboratory exchange flow. As discussed in the next section, computational constraints limited our three-dimensional DNS to $P r=25$ and $R=5$, resulting in slightly different results (figure 1). Although no appreciable changes are seen in the predicted phase speed $c_{r}$ and frequency $\sigma$, there are differences in maximum growth rate and the location of the wavenumber of maximum growth, $k_{\max }$. 

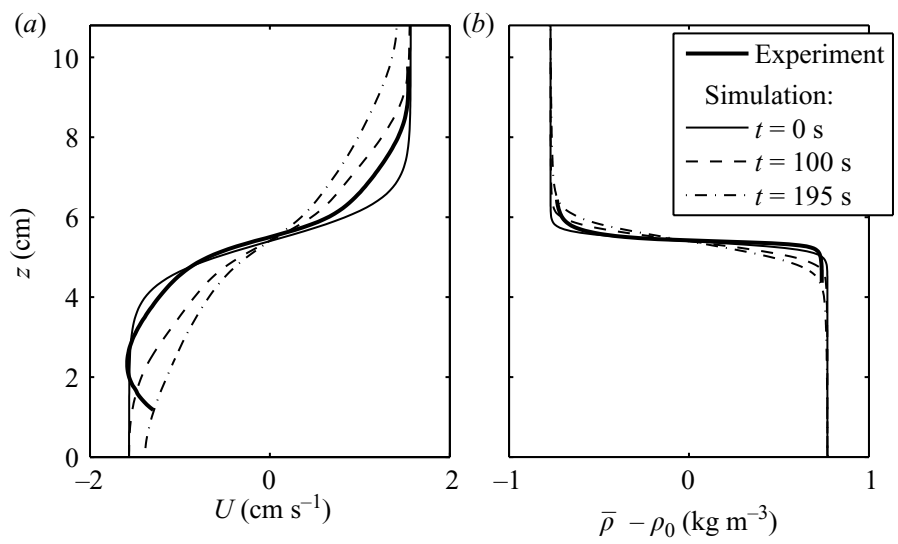

FIGURE 2. Evolution of the background profiles in both the simulation and the experiment: $(a)$ and $(b)$ show temporal changes to the $U(z)$ and $\bar{\rho}(z)-\rho_{0}$ profiles in the simulation with the experimental profiles taken from the channel centre $(x=0)$ in the thick lines.

\section{Methods}

\subsection{Description of the numerical simulations}

The numerical simulations were performed using the DNS code described by Winters, MacKinnon \& Mills (2004), which has been modified to include greater resolution of the density scalar field by Smyth, Nash \& Moum (2005). The simulations were designed to reproduce conditions present in the laboratory experiment as closely as possible, while still conforming to the general methodology used in recent investigations of nonlinear Holmboe waves (Smyth \& Winters 2003; Smyth 2006; Carpenter et al. 2007; Smyth et al. 2007).

The boundary conditions are periodic on the streamwise $(x)$ and transverse $(y)$ boundaries and free-slip on the vertical $(z)$ boundaries. Simulations are initialized with profiles in the form of (2.1) that closely match what is observed in the experiment. Figure 2 shows a sequence of representative $U$ and $\bar{\rho}-\rho_{0}$ profiles at three different times during a simulation, as well as profiles from the experiment for comparison. The periodic boundary conditions of the simulations cause the flow to 'run down' over time; i.e. there is a continual loss of kinetic energy from the shear layer due to viscous dissipation and mixing. This results in an increase of $h$ and $\delta$ over time, as can be seen in figure 2. To indicate conditions at the initial time step ( $t=0 \mathrm{~s})$ of the simulations we will use the subscript ' 0 ' (e.g. $h_{0}$ ).

In order to initiate growth of the primary Holmboe instability, the flow is perturbed with a random velocity field at the first time step. The noise is distributed evenly in the $x, y$ directions but is given greater amplitude near the centre of the shear layer and density interface, in the same manner as Smyth \& Winters (2003). The amplitude of the random perturbation was chosen large enough such that the instability grows to finite amplitude with minimal diffusion of the background profiles, yet it is still small enough that a Courant-Friedrichs-Levy condition for numerical stability is satisfied for the perturbation when superimposed on the mean flow.

While an ideal comparison between simulation and experiment would involve matching all four of the relevant dimensionless parameters, we are constrained by the high computational demands of DNSs. Of particular difficulty is the fine grid resolution required for high- $\operatorname{Pr}$ flows. For this reason we have chosen $\operatorname{Pr}=25$, 


\begin{tabular}{|c|c|c|c|c|c|c|c|}
\hline \multirow[b]{3}{*}{ Experiment } & \multirow{2}{*}{$\begin{array}{l}\text { Resolution } \\
\left\{N_{x}, N_{y}, N_{z}\right\}\end{array}$} & \multicolumn{3}{|c|}{ Parameters } & \multicolumn{2}{|c|}{ Linear theory } & \multirow{2}{*}{$\begin{array}{c}\text { Results } \\
\begin{array}{l}a_{r m s} \\
(\mathrm{~cm})\end{array}\end{array}$} \\
\hline & & $\operatorname{Pr} R$ & $\begin{array}{r}L_{x} \\
R(\mathrm{~cm})\end{array}$ & $\begin{array}{c}L_{y} \\
(\mathrm{~cm})\end{array}$ & $\begin{array}{c}k_{\max } \\
\left(\mathrm{rad} \mathrm{cm}^{-1}\right)\end{array}$ & $\begin{array}{c}c_{r} \\
\left(\mathrm{~cm} \mathrm{~s}^{-1}\right)\end{array}$ & \\
\hline & & 7008 & 8200 & 10 & 0.91 & 0.79 & $\begin{array}{c}0.31 \\
(0.51-0.84)\end{array}$ \\
\hline I Simulation (3D) & $\{3072,128,256\}$ & 255 & $5 \quad 128$ & 5 & 0.79 & 0.84 & 0.62 \\
\hline II Simulation (2D) & $\{3840,1,768\}$ & 7008 & 64 & 0 & 0.91 & 0.79 & 0.48 \\
\hline III Simulation (3D) & $\{1536,256,256\}$ & 255 & 64 & 10 & 0.79 & 0.84 & 0.62 \\
\hline IV Simulation (2D) & $\{1536,1,256\}$ & 255 & $5 \quad 64$ & 0 & 0.79 & 0.84 & 0.74 \\
\hline
\end{tabular}

TABLE 1. Values of various important parameters for the simulations and experiment. The grid point resolution listed for the simulations $\left\{N_{x}, N_{y}, N_{z}\right\}$ is that of the density field. In the case of the three-dimensional (3D) simulations the velocity and pressure fields are resolved on a grid with half the resolution as described in Smyth et al. (2005). The dimensionless parameters listed in the simulations are evaluated using the initial conditions. In all cases we have $J_{0}=0.3, R e_{0}=630$ and $L_{z}=10.8 \mathrm{~cm}$. The dimensional scales can be recovered from $h_{0}=2.0 \mathrm{~cm}$ and $\Delta U=3.1 \mathrm{~cm} \mathrm{~s}^{-1}$. Also included are $k_{\max }$ and $c_{r}$ from the results of the linear stability analysis and the root-mean-square-saturated amplitude observations. The values in parentheses under the experimental $a_{r m s}$ value indicate the predicted range of wave amplitudes once wave stretching is accounted for (see $\S 7$ ). 2D, two dimensional.

opposed to $\operatorname{Pr}=700$ for the laboratory salt stratification. Large values of $R$ also place a high demand on the computational resources, and we have therefore chosen $R_{0}=5$, opposed to the value $R=8$ observed in the experiments. The effects of $\operatorname{Pr}$ and $R$ have been tested by performing a two-dimensional simulation at $\operatorname{Pr}=700$ and $R_{0}=8$. The remaining two parameters, $R e_{0}=630$ and $J_{0}=0.30$, have been matched to the experimental values. Computational constraints also limit the size of the simulation domain. In all cases the vertical depth $L_{z}$ has been matched to the value $10.8 \mathrm{~cm}$ of the experiments. The simulation width $L_{y}=5 \mathrm{~cm}$ has been reduced to half of that in the experiment $\left(L_{y}=10 \mathrm{~cm}\right)$ but was not found to affect the results presented. This reduction in the width of the computational domain enabled a larger length $L_{x}=128 \mathrm{~cm}$, allowing for approximately 16 wavelengths of the most amplified mode, and compares well with $L_{x}=200 \mathrm{~cm}$ in the experiment. In addition to the threeand two-dimensional simulations already mentioned (labelled I and II in table 1, respectively), two supplementary simulations (III and IV) were also performed to test the effects of $L_{y}, R$ and $\mathrm{Pr}$. A summary of the parameters in the experiment and the simulations is shown in table 1 .

It should be noted that in simulations I, III and IV, we have taken $R_{0}=\operatorname{Pr}^{1 / 2}$ in accordance with the scaling of Smyth et al. (1988), as this is applicable to the periodic boundary conditions of the simulations. Indeed, little variation from $R_{0}$ is produced over the duration of these simulations. On the other hand, Hogg \& Ivey (2003) have recognized that a different scaling is expected in exchange flow experiments. Since the shear layer vorticity is advected out of the channel more rapidly than the density interface, the interface is able to accomplish greater diffusion. This leads to the scaling $R \sim \mathrm{Pr}^{1 / 3}$, which is found to be in approximate agreement with $R=8$ observed in the laboratory experiments. Conditions in simulation II have been chosen to match those observed in the experiments and therefore do not follow the $R \sim \operatorname{Pr}^{1 / 2}$ scaling of Smyth et al. (1988). Unless explicitly stated, we will refer to simulation I simply as 'the simulation' hereafter. 

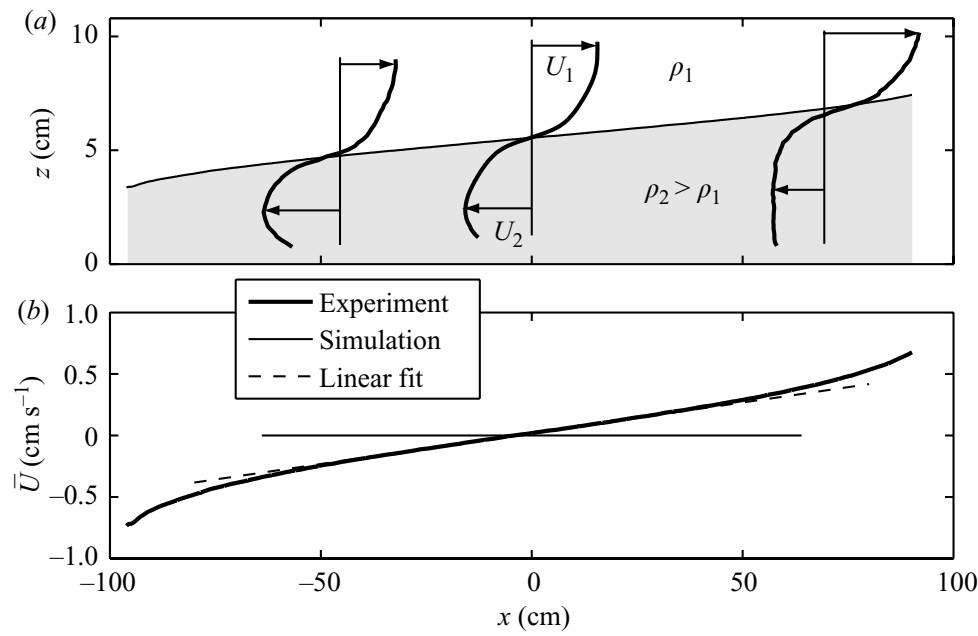

FIGURE 3. Spatial changes in $U(z)$ and layer depths that occur along the laboratory channel are shown in $(a)$, with the corresponding distribution of $\bar{U}(x)$ in $(b)$. A linear fit to $\bar{U}(x)$ in the central portion of the channel is shown as the dashed line, and the mean velocity in the simulation domain is given by the thin solid line.

\subsection{Description of the laboratory experiments}

The laboratory experiments were performed in the exchange flow facility described by Tedford et al. (2009b). A complete discussion of the experimental procedures and apparatus can be found in that study; however, we now provide a summary of the pertinent features.

The apparatus consists of two reservoirs connected by a rectangular channel $200 \mathrm{~cm}$ in length and $10 \mathrm{~cm}$ in width. The reservoirs are initially filled with fresh and saline water $\left(\Delta \rho=1.41 \mathrm{~kg} \mathrm{~m}^{-3}\right)$ such that the depth in the channel is $10.8 \mathrm{~cm}$. A bidirectional exchange flow is initiated by the removal of a gate from the centre of the channel. After an initial transient period in which gravity currents propagate to each reservoir and mixed interfacial fluid is advected from the channel, the flow enters a period of steady exchange where the density interface is found to display an abundance of Holmboe wave activity. In contrast with the rundown conditions in the DNS, the storage of unmixed water in the reservoirs maintains a steady exchange flow for approximately $600 \mathrm{~s}$. Our comparison is restricted to instabilities observed during the period of steady exchange from a series of replicated experiments.

The exchange flow exhibits internal hydraulic controls at the entrance to each of the reservoirs, effectively isolating the channel from disturbances in the reservoirs and enforcing radiation boundary conditions at the channel ends. Friction between the layers leads to a gradually sloping density interface (figure 3 ) that produces an $x$-dependent mean velocity $\bar{U}=\left(U_{1}+U_{2}\right) / 2$, with the upper $\left(U_{1}\right)$ and lower $\left(U_{2}\right)$ layer velocities defined as the maximum and minimum free-stream velocities (Tedford et al. $2009 b)$. The gradual variation of $\bar{U}(x)$ along the laboratory channel is a result of the acceleration in each of the layers owing to the sloping interface. This variation is shown in figure $3(b)$ and is found to be a near-linear function of $x$ for the central portion of the channel. In contrast, $\bar{U}$ is identically zero throughout the domain in the simulation, because of the periodic boundary conditions. This difference in mean flow is found to have important effects on the nonlinear development of the Holmboe wave field. 


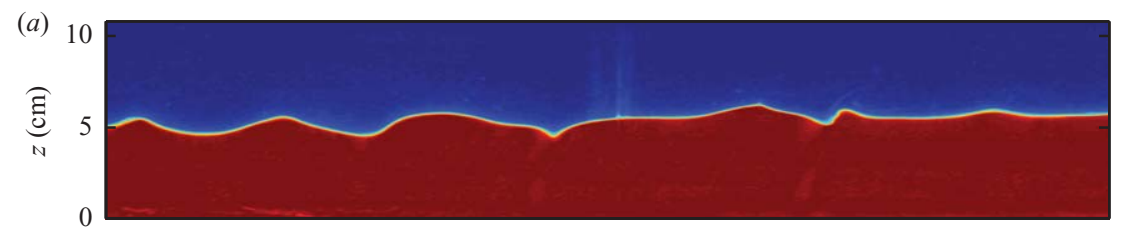

Experiment

Three-dimensional

$\operatorname{Pr}=700$

$R=8$

(b)

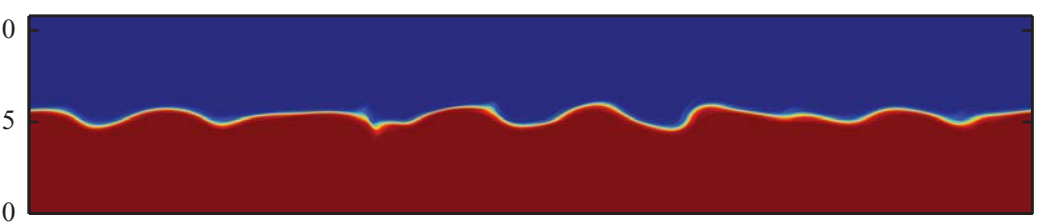

Simulation II

Two-dimensional

$P r=700$

$R_{0}=8$

$t=78 \mathrm{~s}$

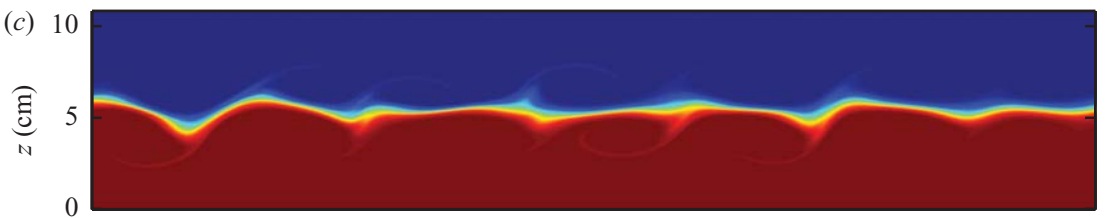

Simulation I

Three-dimensional

$P r=25$

$R_{0}=5$

$t=75 \mathrm{~s}$

(d)

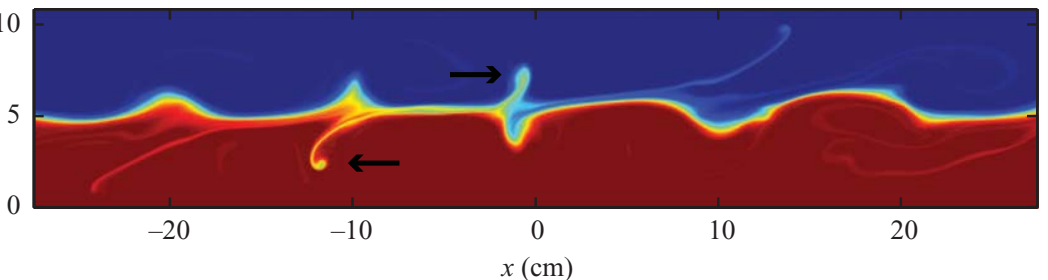

Simulation I

Three-dimensional

$P r=25$

$R_{0}=5$

$t=98 \mathrm{~s}$

Figure 4. Representative plots of the density field for $(a)$ the experiment along with $(b)$ simulation II and $(c, d)$ simulation I. The plot in $(d)$ is taken at a later time when two ejections are underway, indicated by the arrows. The $x$-axis has been shifted by $L_{x} / 2$ to the left in $(d)$ to better display the ejection process.

\section{Wave structure}

In the first instance, it is beneficial to perform a simple visual comparison of the density structure of the waves. This is shown in figure 4, where a representative photograph of the laboratory waves is displayed above plots of the density field from the two-dimensional simulation, simulation II (figure $4 b$ ), and the three-dimensional simulation, simulation I (figure $4 c, d$ ). The density structure in figure $4(a, b)$ is very similar, as each initially has an identical set of dimensionless parameters, differing only in the initial and boundary conditions. However, as the flow evolves, inevitable differences in $R, J$ and $R e$ occur because of the growth of $h$ and $\delta$ in time. In all panels of figure 4 it can be seen that although many of the waves display the typical form of the Holmboe instability and consist of cusps projecting into the upper and lower layers, others appear sinusoidal in form. The waves focused above the density interface (upward-pointing cusps) are moving from left to right, in the same direction as the flow in the upper layer, while the waves in the lower layer (downward cusps) move at an equal but opposite speed with respect to the mean velocity.

An important feature of nonlinear Holmboe waves is the occasional ejection of stratified fluid from the wave crests into the upper and lower layers. Two such ejections are shown in figure $4(d)$ where indicated and can be characterized by thin wisps of fluid being drawn from the wave crest and advected by the mean flow. These wisps often settle back to the interface level, contributing to the accumulation of the mixed 
(a) Simulation

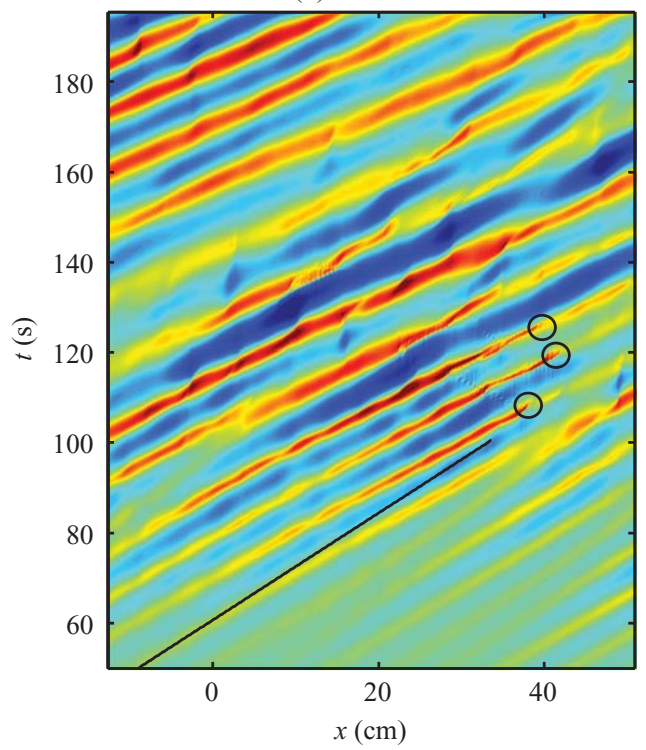

(b) Experiment

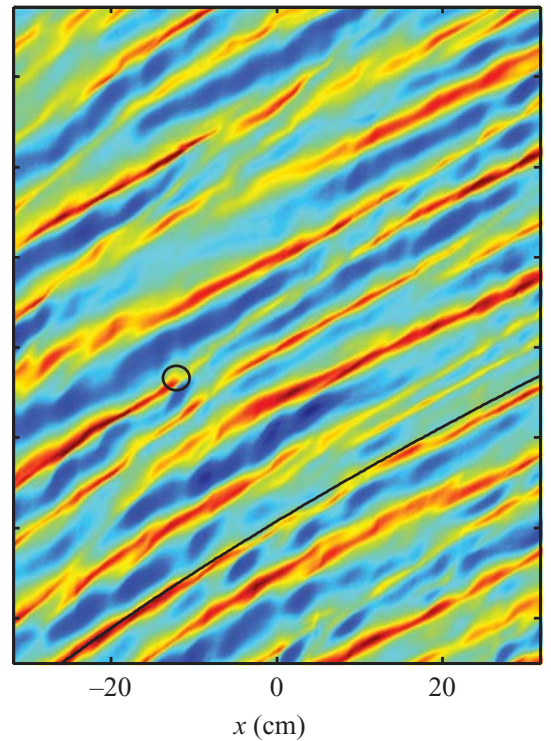

FIGURE 5. Rightward-propagating wave characteristics for $(a)$ the simulation and $(b)$ the experiment. Shading represents the elevation of the density interface, with red indicating a high (crest) and blue indicating a low (trough), and has a different colour scale in both $(a)$ and $(b)$. The solid black lines indicate the characteristic slope given by the linear prediction of phase speed $c_{r}$. In the case of the laboratory experiment, $c_{r}$ has a slight curvature, since the changes in $\bar{U}$ along the channel have been included. The dark circles indicate locations and times of ejections.

fluid there. This accumulation is observed to a much greater extent in figure 4(c, $d$ ) and should be expected because of the larger value of $R_{0}$, as well as the higher diffusion that comes with the lower $P r$ used in this simulation. Although ejections are observed in both the laboratory experiment and the high- $P r$ and high- $R$ simulation (II), there is a greater frequency of occurrence in the lower- $\operatorname{Pr}(\operatorname{Pr}=25)$ and lower- $R$ $(R=5)$ simulation $(\mathrm{I})$.

Holmboe's instability has the uncommon property that under certain conditions, the growth of the primary instability may take place as a three-dimensional wave. Such a wave would travel oblique to the orientation of the shear and produces significant departures from a two-dimensional wave. One of the conditions for this three-dimensional growth is that $R e$ should be sufficiently low (Smyth \& Peltier 1990). As the laboratory experiments are carried out at low Re and show some variation in the spanwise direction, it must be questioned whether the growth of the primary Holmboe instability is three-dimensional. This is easily tested by the simulation results, which show a clear two-dimensional growth (see $\S 7$ as well), even to an initially random perturbation as described above. We can therefore confirm that the primary instability is two-dimensional for the conditions examined in the present study.

\section{Phase speed}

Many of the basic features in the wave field are revealed by an $x-t$ characteristic diagram of the density interface elevation, shown in figure 5 for both the simulation 
and the experiment. Although the interface consists of contributions from both upper and lower Holmboe wave modes (each travelling in opposite directions), we have filtered the characteristics to reveal only the upper, rightward-propagating wave modes. This is done by performing a two-dimensional Fourier transform to express the interface elevation in the wavenumber-frequency $(k, \omega)$ plane. The leftwardpropagating waves, for example, are then removed by setting the quadrants in which $k$ and $\omega$ have the same sign to zero and performing the inverse transform. The same procedure is applied to the opposite quadrants to remove the rightward-propagating waves.

Certain differences between the simulation characteristics (figure $5 a$ ) and the experimental characteristics (figure $5 b$ ) are immediately apparent. The experimental characteristics exhibit a greater degree of irregularity. Since each plot represents a two-dimensional slice from a three-dimensional field, this may be a result of greater variability in the transverse direction in the case of the experiments. Since the waves in the simulation develop from an initial random perturbation at $t=0$, there is also a temporal growth of the average wave amplitude in figure 5(a) that is not present in the experimental characteristics.

Despite these apparent differences in the characteristics, the phase speeds (given by the slope of the characteristics) are in good agreement. The observed phase speeds in both the simulation and the experiment are found to be slightly greater than the predictions of linear theory (solid lines), which has been noted in previous studies (Haigh 1995; Hogg \& Ivey 2003; Tedford et al. 2009b). However, the observations also suggest an increase in phase speed with wave amplitude. This is a quintessential feature of nonlinear wave behaviour (e.g. Stokes waves). Note that relatively highfrequency oscillations in wave amplitude and phase speed are present in both sets of characteristics in figure 5, which appear as a 'pulsing' of the characteristics. This is a well-known feature of Holmboe waves due to the interaction between the two oppositely propagating modes (Smyth et al. 1988; Zhu \& Lawrence 2001; Hogg \& Ivey 2003).

Sudden decreases in wave amplitude can be seen in both sets of characteristics at a number of times and locations. It is often the case that these sudden amplitude changes are a result of the ejection process. Instances in which ejections occur have been identified in figure 5 and are denoted by the circles. It is generally observed that the ejection process preferentially acts on the waves with the largest amplitude, similar to the breaking of surface waves (Longuet-Higgins \& Cokelet 1978; LonguetHiggins \& Dommermuth 1997).

\section{Spectral evolution}

This section concerns the distribution and evolution of wave energy with $k$. It will be shown that there are two different processes acting separately in the simulation and experiment that are responsible for a shifting of wave energy to lower $k$ (i.e. longer waves).

\subsection{Frequency shifting}

In order to gain an understanding of the wave spectrum, it is first useful to carefully examine the characteristic diagrams. Figure 6 shows rightward-propagating characteristics from both the simulation and the experiment that highlight the location of wave crests (in white) and troughs (in grey). Characteristics from the simulation (figure $6 a$ ) are discussed first and are shown for the entire computational domain. 
(a) Simulation

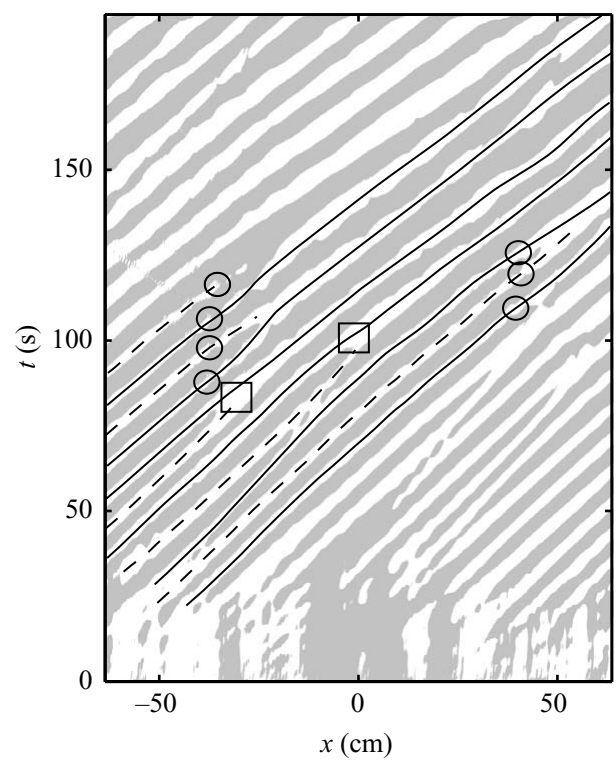

(b) Experiment

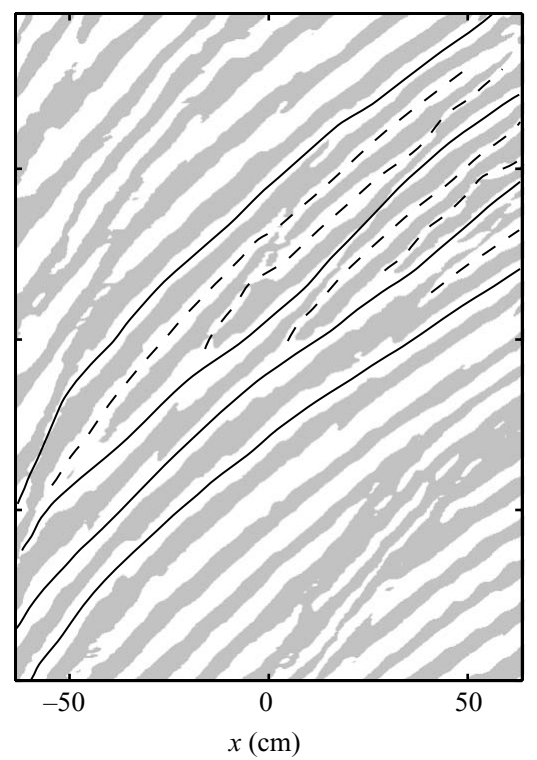

FIGURE 6. Rightward-propagating wave characteristics for $(a)$ the simulation and $(b)$ the experiment $(b)$. White indicates a wave crest, while grey indicates a wave trough. In each panel a number of wave crests are indicated by the solid and dashed lines. In $(a)$, the dashed lines correspond to waves that are 'lost' over the duration of the simulation, whereas in $(b)$, the dashed lines correspond to waves that have formed within the channel. Only the central portion of the laboratory channel corresponding to the simulation domain has been shown. The circles and squares indicate locations and times of ejections and pairing events, respectively. It should be noted that characteristics from a different experiment than those shown in figure $5(b)$ have been used here in $(b)$ to best illustrate the wave formation process.

Beginning with the initial random perturbation at $t=0$, energy is extracted from the mean flow by the instability and fed into the wave field at, or very close to, the wavenumber of maximum growth, $k_{\max }$. This results in approximately 16 waves in the computational domain (given by $L_{x} k_{\max } / 2 \pi$ ) for early times. We see however that as the simulation proceeds wave crests are continually being 'lost' over time. This feature is highlighted by the solid and dashed lines that are used to trace the wave crests in figure 6(a). The dashed lines indicate wave crests that are 'lost', while the solid lines correspond to crests that persist. This process of losing waves results in an observed frequency $\omega$ that is continually shifted downwards. It has recently been noted in Holmboe wave simulations by Alexakis (2009) but has not been described in detail. This 'frequency downshifting' or 'wave coarsening' has, however, been noted previously in other nonlinear wave systems (e.g. Huang, Shen \& Long 1999; Balmforth \& Mandre 2004).

In the simulations performed, the ejection process typically results in a loss of waves and a downshift in frequency (figure $6 a$ ). This observation mirrors similar findings in the frequency downshifting of nonlinear surface gravity waves, where the occurrence of wave breaking is related to lost waves (Huang, Long \& Shen 1996; Tulin \& Waseda 1999).

Close examination of the vorticity field also suggests that the Holmboe waves undergo a vortex pairing process. Each interfacial wave is led by a vortex formed from the 'rolling up' of the initial shear layer vorticity. This leading vortex propagates 

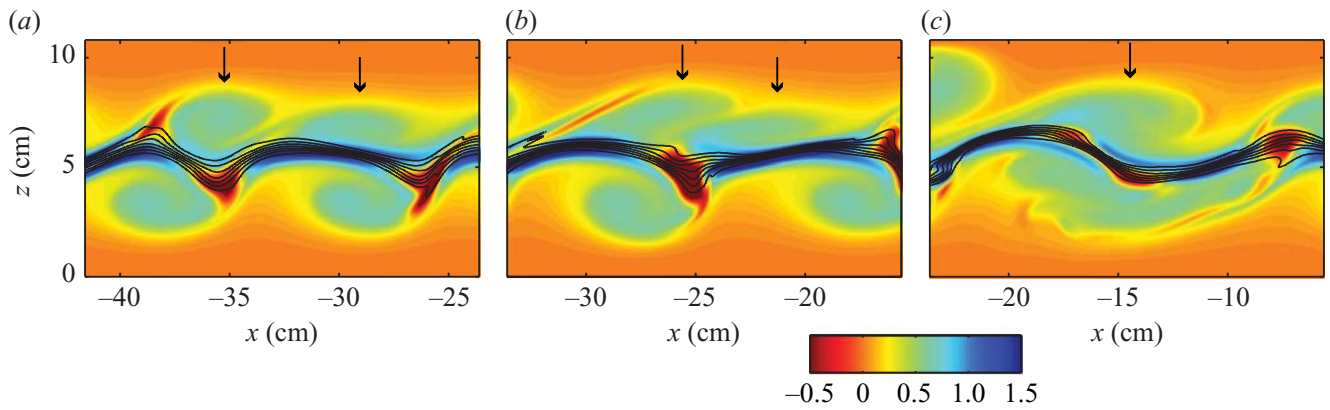

FIGURE 7. Vorticity field from the simulation illustrating the vortex pairing process. Only the spanwise $(y)$ component of the vorticity vector is shown for the times $(a) t=77 \mathrm{~s},(b) t=87 \mathrm{~s}$ and (c) $t=97 \mathrm{~s}$. The black contours denote isopycnals with a spacing of $\Delta \rho / 8$, and the arrows indicate the position of vortices in the upper layer that undergo pairing. Note that two vortices are pairing in the lower layer in $(c)$ as well. The colour bar is in units of $\Delta U / h_{0}$.

with the waves in the upper and lower layers and can be seen in the vorticity field plotted in figure $7(a)$. The pairing process is seen to act on the two vortices (indicated by the arrows) in the upper layer of figure 7; they draw closer together, rotating about each other (figure $7 b$ ), and eventually merge into a single vortex (figure $7 c$ ). Although the pairing of adjacent vortices is a well-known feature of homogeneous and weakly stratified shear layers (Browand \& Winant 1973), it has received little attention in Holmboe waves, the only observation being that of Lawrence, Browand \& Redekopp (1991). This is an additional means to effect a shift of wave frequency and is denoted by the square symbols in figure $6(a)$.

In contrast, figure $6(b)$ shows that the experimental characteristics display a distinctly different behaviour. In this case, new wave crests are continually being formed as the waves traverse the channel. Again, this process is highlighted by the tracing of crests by the solid and dashed lines. Now, the dashed lines represent new wave crests that have been formed within the channel. This process results in an increasing $\omega$ with $x$ in the experiments.

Tedford et al. (2009b) have explained the formation of new waves as follows. As waves propagate through the channel they are accelerated by the increasing mean velocity $\bar{U}(x)$. This leads to a 'stretching' of the waves that decreases $k$ from near $k_{\max }$, where the waves initially formed, to lower values (i.e. longer wavelengths). Once a sufficiently low $k$ is achieved, the Holmboe instability mechanism acts between the wave crests to form additional waves. This feeds energy back into the wave field near $k_{\max }$, resulting in an average $k$ that is constant across the channel and an increasing $\omega$.

The two processes, namely wave coarsening in the simulation and wave stretching in the experiments, can be described quantitatively using wave spectra.

\subsection{Wave energy spectra}

Differences between the processes responsible for modifying $k$ in both the simulation and the experiment can be seen in figure 8. It demonstrates how wave energy (indicated by the dark bands) is redistributed in $k$ over time.

The density interface elevation spectrum of the simulation (figure $8 a$ ), which has been normalized by the variance in order to remove the time-dependent growth of the waves, shows a discrete transfer of wave energy to lower $k$. The simulation spectrum is required to evolve in discrete steps because of the periodic boundary conditions 


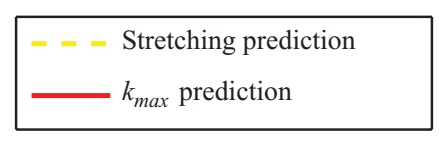

(a) Simulation

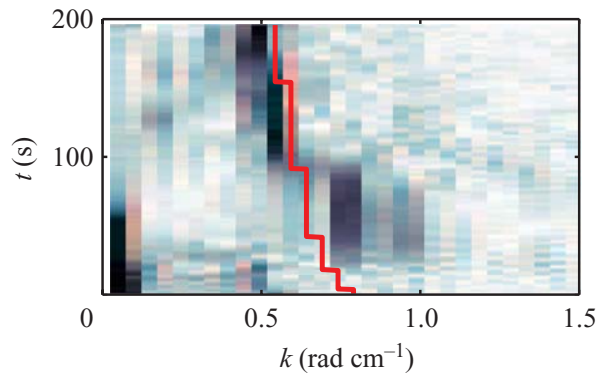

(b) Experiment

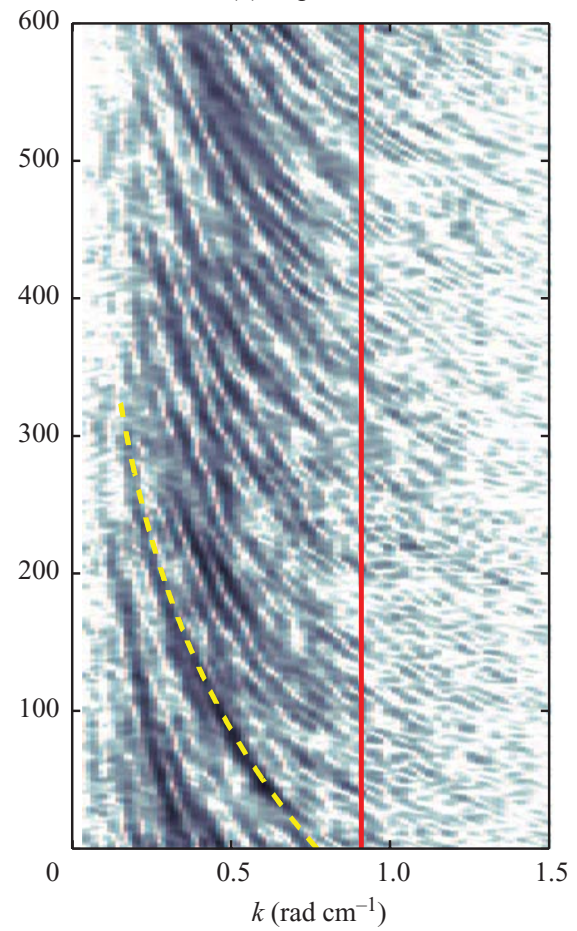

FIGURE 8. Spectral evolution of the rightward-propagating waves from $(a)$ the simulation and (b) the experiment. Dark colours denote a high in energy which is proportional to the mean square amplitude of the interface displacement. The wave energy has been normalized by the variance of the interface elevation in $(a)$ to remove the time-dependent wave growth. Linear stability theory is used to predict $k_{\max }$ (red lines), which changes in time for the simulations. The predicted stretching of wave energy in the experiment by $\bar{U}(x)$ to lower $k$ is shown as the yellow dashed line in $(b)$. The beginning of the steady period of exchange is referenced to $t=0 \mathrm{~s}$ in $(b)$.

(i.e. in wavenumber increments of $\Delta k=2 \pi / L_{x}$ ). As a point of comparison, the $k_{\text {max }}$ prediction from linear stability theory is plotted in red. The predicted $k_{\max }$ has been discretized according to the boundary conditions and decreases in time because of the diffusion of the background profiles, i.e. the increase in the shear layer thickness $h(t)$.

The spectral evolution plot (figure $8 a$ ) compliments the characteristic diagram of figure $6(a)$, showing an initial input of energy at $k_{\max }(t=0)$ and a subsequent shifting of that energy to lower $k$. It is interesting to note that the $k_{\max }(t)$ curve shows the same general trend as the concentration of wave energy (shown by the dark 'blocks' in figure $8 a$ ). Although the details are unclear, we speculate that the shift in wave energy to lower $k$ is the result of nonlinear processes such as the ejections and vortex pairing.

It is apparent from the wave spectra in figure $8(b)$ that the process responsible for the redistribution of wave energy in the experiments is a continuous one. Energy at any given time is found to be focused in a number of 'bands'. These bands originate near $k_{\max }$ and move towards lower $k$ in time. In addition, they all appear to have a similar trajectory in $k t$-space. Tedford et al. (2009b) hypothesized that these bands 
are a result of the stretching of wave energy to lower $k$ by $\bar{U}(x)$. We now formulate a simple model in order to quantify this hypothesis.

\subsubsection{Wave stretching prediction}

The changes in $k$ that result from wave stretching by $\bar{U}(x)$ can be described by an application of gradually varying wave theory. This theory assumes that the density interface elevation $\eta(x, t)$ may be expressed in terms of a gradually varying amplitude $a(x, t)$ and a rapidly varying sinusoidal component, viz.

$$
\eta(x, t)=\operatorname{Re}\left\{a(x, t) \mathrm{e}^{\mathrm{i} \theta(x, t)}\right\},
$$

so that it is possible to define a local wavenumber and frequency in terms of the phase function $\theta(x, t)$ by $k \equiv \partial \theta / \partial x$ and $\omega \equiv-\partial \theta / \partial t$, respectively. These definitions of $k$ and $\omega$ lead directly to the conservation of waves relation

$$
\frac{\partial k}{\partial t}+\frac{\partial \omega}{\partial x}=0
$$

by Clairaut's theorem, assuming that $\theta(x, t)$ is sufficiently differentiable. Recognizing that $\omega$, which is the frequency that a stationary observer would measure, includes both an intrinsic portion $\sigma(k)$ and an advective portion $k \bar{U}$ leads to

$$
\omega=\sigma(k)+k \bar{U}(x) \text {. }
$$

Substituting into (6.2) gives

$$
\frac{\mathrm{D} k}{\mathrm{D} t}=-S k
$$

where the material derivative, defined as

$$
\frac{\mathrm{D}}{\mathrm{D} t} \equiv \frac{\partial}{\partial t}+\left(c_{g}+\bar{U}\right) \frac{\partial}{\partial x}
$$

denotes changes in time while moving at the speed $c_{g}+\bar{U}$, and $c_{g} \equiv \mathrm{d} \sigma / \mathrm{d} k$ is the intrinsic group speed. Wave energy is expected to propagate through the channel at the speed $c_{g}+\bar{U}$. We have also defined $S \equiv \mathrm{d} \bar{U} / \mathrm{d} x$, which is found to be very nearly constant in the central portion of the laboratory channel (see figure $3 b$ ). Choosing a Lagrangian frame of reference, which moves at the speed $c_{g}+\bar{U}$ through the channel, allows for a simple integration of (6.4) to give

$$
k(t)=k_{*} \mathrm{e}^{-S\left(t-t_{*}\right)},
$$

where $k_{*}=k\left(t_{*}\right)$ is some initial value of $k$ that wave energy begins the stretching process at. A referee has pointed out that a simple (though equivalent) way of arriving at the result (6.5) is to consider two crests a distance $\lambda$ apart. Since these crests are in a stretching velocity field $\bar{U}(x)=S x$, they move apart at a rate $\mathrm{d} \lambda / \mathrm{d} t=\bar{U}(x+\lambda)-$ $\bar{U}(x)=S \lambda$. From this it follows that $\lambda(t)=\lambda_{*} \mathrm{e}^{S\left(t-t_{*}\right)}$, or in terms of wavenumber $k$, we recover (6.5).

By direct measurement of $S$ in the laboratory channel, a comparison is now possible between the prediction of (6.5) and the bands of energy in the observed spectral evolution. The prediction is shown by the yellow dashed line in figure $8(b)$ and is found to be in agreement with the observations. The largest source of error in the spectral prediction of (6.5) comes from the assumption that $S$ is constant. A high level of certainty in the value of $S$ has been obtained because of averaging over many experiments (see Tedford et al. $2009 \mathrm{~b}$ for further details). The close agreement between the predictions of the simple wave stretching model and the experimental 
observations validates the hypothesis that the spectral shift towards lower $k$ is a result of wave stretching. It also reveals that our assumption of wave conservation is justified. This is not in contradiction with the formation of new waves described in $\S 6.1$, since wave conservation is applied only after energy is fed into the wave field by the instability mechanism.

The spectral evolution of the waves observed in the laboratory experiment suggest the following interpretation. The instability mechanism draws energy into the Holmboe wave field near $k_{\max }$ in accordance with the predictions of linear theory. The waves then grow relatively rapidly and uniformly throughout the channel; however, once they have formed they are subjected to a mean flow that stretches them to longer wavelengths and lower $k$ as described by (6.5). After a sufficient decrease in $k$ the linear instability acts to introduce energy back into the wave field near $k_{\max }$, thus initiating another of the 'bands' in figure $8(b)$. Once a new band of wave energy has formed, its subsequent evolution is governed by the conservation of waves.

The vortex pairing and ejections that led to wave coarsening in the simulations are not expected to play an important role in the spectral evolution of the laboratory experiments. This is due to two different effects of the wave stretching: first, it will tend to inhibit vortex pairing by increasing the distance between vortices, and second, it will reduce the occurrence of ejections by decreasing wave amplitudes, as we now discuss.

\section{Wave growth and amplitude}

The final basic parameter that we intend to compare is the wave amplitude $a$. This feature of the wave field is determined when the linear growth reaches some level at which it saturates. It is a nonlinear property of the waves and may involve three-dimensional effects as well as interaction with the mean flow. We begin by discussing the various phases of wave growth.

\subsection{Wave growth}

In the simulation, the instability mechanism causes the growth of waves from an initial random perturbation into a large-amplitude nonlinear wave form. This growth process is best illustrated by considering the kinetic energy of the waves, $\mathscr{K}$. Following Caulfield \& Peltier (2000), we partition $\mathscr{K}$ into a two-dimensional kinetic energy $\mathscr{K}_{2 d}$ associated with the primary Holmboe wave and a three-dimensional component $\mathscr{K}_{3 d}$ that provides a measure of the departures from a strictly two-dimensional wave. By this partitioning we have

$$
\mathscr{K}=\mathscr{K}_{2 d}+\mathscr{K}_{3 d},
$$

where

$$
\mathscr{K}_{2 d}=\left\langle\boldsymbol{u}_{2 d} \cdot \boldsymbol{u}_{2 d} / 2 \mathscr{K}_{0}\right\rangle_{x z} \quad \text { and } \quad \mathscr{K}_{3 d}=\left\langle\boldsymbol{u}_{3 d} \cdot \boldsymbol{u}_{3 d} / 2 \mathscr{K}_{0}\right\rangle_{x y z} \text {, }
$$

and we have used

$$
\begin{aligned}
\boldsymbol{u}_{1 d}(z, t) & =\langle\boldsymbol{u}\rangle_{x y}, \\
\boldsymbol{u}_{2 d}(x, z, t) & =\left\langle\boldsymbol{u}-\boldsymbol{u}_{1 d}\right\rangle_{y}, \\
\boldsymbol{u}_{3 d}(x, y, z, t) & =\boldsymbol{u}-\boldsymbol{u}_{1 d}-\boldsymbol{u}_{2 d},
\end{aligned}
$$

with $\langle\cdot\rangle_{i}$ representing an average in the direction $i$ and $\mathscr{K}_{0}$ the total kinetic energy at $t=0$.

The $\mathscr{K}_{2 d}$ and $\mathscr{K}_{3 d}$ components are plotted on a log scale in figure 9 for the simulation. The plot indicates that after a start-up period in which the energy of 


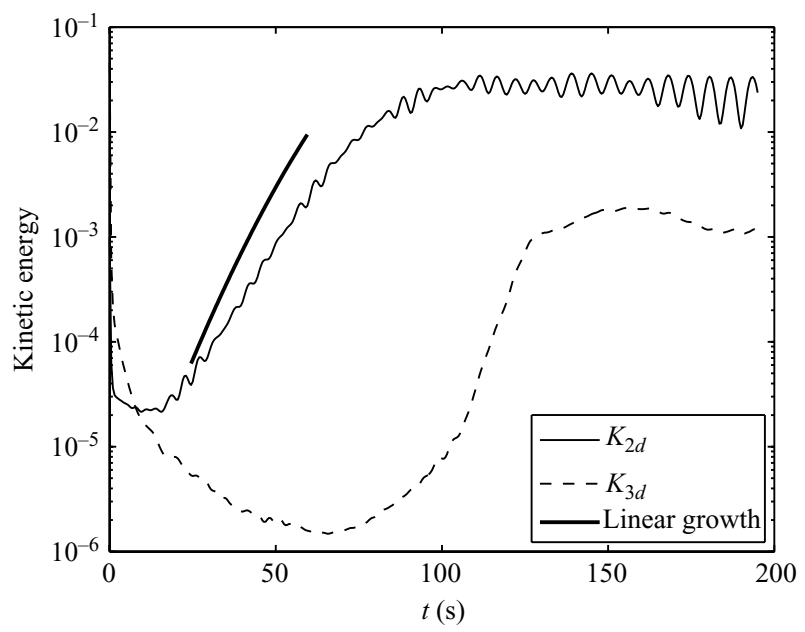

Figure 9. Growth of $\mathscr{K}_{2 d}$ and $\mathscr{K}_{3 d}$ for the simulation. The slope of the thick line gives the linear growth rate prediction of $\mathscr{K}_{2 d}$, which is a function of time owing to the changing background profiles.

the initial perturbation rapidly decays, a stage of exponential growth is achieved in $\mathscr{K}_{2 d}$. This stage of exponential growth can be compared with the prediction of linear theory and is found to be less than the prediction $\left(k c_{i}=0.10 \mathrm{~s}^{-1}\right.$ versus the observed $k c_{i} \approx 0.06 \mathrm{~s}^{-1}$ ). One possible explanation of this discrepancy in $k c_{i}$ is due to the fairly rapid spreading rate of the shear layer, measured by $r=h^{-1} \mathrm{~d} h / \mathrm{d} t$, during the stage of linear growth up to $t \approx 65 \mathrm{~s}$. This spreading is due to the viscous diffusion of vorticity in the shear layer and may be compared with the growth rate of the instability by the ratio $r / k c_{i}$. Linear theory implicitly assumes that $r / k c_{i} \ll 1$, indicating that there is no time dependence of the background profile. While $r / k c_{i} \approx 0.1$ is relatively small during the linear growth period, it may be sufficient to explain the differences in observed growth rates.

The wave growth is entirely two-dimensional until the waves have reached a finite amplitude $(t \approx 65 \mathrm{~s})$, at which point the growth of three-dimensional secondary structures begins (see Smyth 2006 for a discussion of this process in Holmboe waves). However, the waves remain primarily two-dimensional throughout the simulation, with $\mathscr{K}_{3 d}$ at least an order of magnitude smaller than $\mathscr{K}_{2 d}$. There is not a welldefined transition to turbulence, as is found in other studies of stratified shear layers (e.g. Caulfield \& Peltier 2000; Smyth, Moum \& Caldwell 2001), probably due to the low $R e$. Once the saturated amplitude is reached, there is a slow decline of $\mathscr{K}$ over the remainder of the simulation.

In the laboratory experiments we have focused only on the period of steady exchange and therefore do not observe a time-dependent growth of the wave field on average. However, as discussed previously, the instability is constantly acting to produce new waves along the channel. It is difficult to measure the growth rate of these waves, but they appear to reach a saturated amplitude rapidly, suggesting that they are strongly forced by disturbances within the channel.

\subsection{Comparison of saturated amplitudes}

Although the transient growth of the instability is difficult to quantify in the experiments, it is possible to measure the mean amplitude of the waves. This is 
done by using the root-mean-square amplitude of the interface elevation $\eta(x, t)$, given by

$$
a_{r m s}(x)=\sqrt{\frac{1}{T} \int_{T} \eta^{2} \mathrm{~d} t},
$$

where $T$ denotes the duration of the steady period of exchange. When averaged over a number of experiments $a_{r m s}$ is found to display little dependence on $x$. A similar $a_{r m s}$ can be defined for the simulations; however, the temporal average is replaced by a spatial average in $x$. The growth of $a_{r m s}$ in time in the simulations shows a behaviour to similar $\mathscr{K}_{2 d}$ : an exponential initial growth, followed by a saturation and subsequent decay. The saturated (maximum) amplitude reached during each of the simulations is shown in table 1 , along with the mean amplitude in the experiments.

The first feature to note is that the waves of the two-dimensional simulation (II) at $R_{0}=8$ and $\mathrm{Pr}=700$ (matching the conditions in the experiment) have a lower amplitude than all the other simulations, especially the two-dimensional simulation (IV) at $R_{0}=5, \operatorname{Pr}=25$. This indicates that there is a possible dependence of the saturated amplitude on $R, P r$. Most importantly, the amplitude measured in the experiments is significantly smaller than any of the saturated amplitudes reached in the simulations. The small amplitudes observed in the experiments can be explained by, once again, appealing to the effects of wave stretching.

\subsubsection{Wave stretching effects on amplitude}

To understand the effects of wave stretching on amplitude in the experiments, we apply principles that have been established for waves on slowly varying currents (e.g. Peregrine 1976). In doing so, we assume that the Holmboe waves may be represented by a simple train of linear internal waves that satisfy the dispersion relation in figure $1(b)$. We are then able to track the changes in wave amplitude that occur as a result of the spatially varying mean velocity $\bar{U}(x)$, i.e. the wave stretching. In this simplified model, the total wave energy is not conserved, since there is an exchange between the mean flow and the waves. However, if the spatial scale of variation of the mean flow $\bar{U}(x)$ is large compared with the wavelength, it can then be shown that wave action is conserved (see Bretherton \& Garrett 1969 for a full discussion). This is given as $E / \sigma$, where $E$ is the wave energy density, and recall that $\sigma(k)$ is the intrinsic wave frequency. Substitution into the conservation law and following a similar procedure to $\S 6.2 .1$ leads to a similar result,

$$
\frac{\mathrm{D}}{\mathrm{D} t}\left(\frac{E}{\sigma}\right)=-S\left(\frac{E}{\sigma}\right),
$$

which describes changes in action density due to the stretching by $\bar{U}$. In arriving at (7.5) we have neglected a term that is proportional to $\mathrm{d}^{2} \sigma / \mathrm{d} k^{2}$, which is small in the range of $k$ that we are interested in (see figure $1 b$ ). Taking $S$ to be constant once again allows for simple integration of (7.5) to give

$$
\left(\frac{E}{\sigma}\right)=\left(\frac{E}{\sigma}\right)_{*} \mathrm{e}^{-S\left(t-t_{*}\right)}
$$

For linear internal waves $E \propto a^{2}$, so that we have an estimate of the reduction in wave amplitude due to stretching of

$$
\frac{a}{a_{*}}=\sqrt{\frac{\sigma}{\sigma_{*}}} \mathrm{e}^{-S\left(t-t_{*}\right) / 2} .
$$


If we now take the intrinsic frequency $\sigma \propto k$, as suggested by the linear dispersion relation in figure $1(b)$, and use $(6.5)$ we obtain

$$
\frac{a}{a_{*}}=\mathrm{e}^{-S\left(t-t_{*}\right)} .
$$

By inspection of figure $8(b)$, we can estimate a time interval $\Delta t$ that wave energy spends in the channel (i.e. the average time interval that the dark bands appear for) to be between 100 and $200 \mathrm{~s}$. The amplitude reduction is therefore in the range $0.37<\mathrm{e}^{-S \Delta t}<0.61$.

Given this reduction and assuming that no other processes are taking place that may affect the wave amplitudes, we would expect amplitudes in the range of that shown in table 1 , given in parentheses. This adjusted amplitude is comparable to the observations in the simulations and demonstrates that - in the absence of other processes - the stretching of wave action is significant in reducing the experimental wave amplitudes.

\section{Conclusions}

We have compared simulations of Holmboe wave fields with the results of the laboratory experiments. A meaningful comparison was possible, since both methods exhibit only gradual variations in the mean flow. In the laboratory experiment, the mean flow is spatially varying, whereas the numerical simulations display a temporal variation. Focusing on basic descriptors of the waves, such as phase speed, wavenumber and amplitude, we have identified a number of processes affecting the nonlinear behaviour of Holmboe wave fields.

Similarities between the results of the two methods include the basic structure of the waves and the phase speeds. The observations show slightly greater phase speeds when compared with the predictions of linear theory, in agreement with previous studies (Haigh 1995; Hogg \& Ivey 2003; Tedford et al. 2009b). Further departures from the linear predictions are attributed to a nonlinear dependence of the phase speed on amplitude.

The greatest differences between simulation and experiment are found in the spectral evolution and wave amplitudes. In simulations, a transfer of wave energy to lower $k$ was found to result from wave coarsening, which caused waves to be 'lost' through discrete merging events. These events were typically found to result from both vortex pairing and the ejection process, the latter of which is suggested to be similar to wave breaking in surface waves, since it appears to act preferentially to reduce the amplitude of the largest (steepest) waves. A detailed investigation of both ejections and vortex pairing in Holmboe waves is currently underway.

The shift of wave energy to lower $k$ that was observed in the experiments can be attributed to the 'stretching' of the wave field by the spatially accelerating mean flow. This suggestion of Tedford et al. (2009b) has been confirmed by a simple application of gradually varying wave theory, which is able to predict the time dependence of the spectral shift.

The wave stretching process is also expected to have a significant effect in reducing the wave amplitudes observed in the experiments. This conclusion appears sufficient to explain discrepancies between wave amplitudes in experiment and simulation and is based on a simple application of the conservation of wave action. In this application we have assumed that no other processes are actively influencing the wave amplitude; however, a dependence of wave amplitude on $R$ and $\operatorname{Pr}$ has been noted. 
Our comparisons of the simulations and the experiments reveal ways in which the nonlinear evolution of a Holmboe wave field is dependent on the mean flow and hence on the boundary conditions. This must be considered when studying the nonlinear behaviour of the Holmboe instability.

We would like to thank Neil Balmforth for helpful discussions and Bill Smyth for providing the code for the linear stability analysis and the simulations. Funding and support provided by the Natural Sciences and Engineering Research Council of Canada and the Canada Research Chairs Program is gratefully acknowledged. The computational resources required for this study were provided by WestGrid.

\section{REFERENCES}

AleXakis, A. 2005 On Holmboe's instability for smooth shear and density profiles. Phys. Fluids 17, 084103.

Alexakis, A. 2007 Marginally unstable Holmboe modes. Phys. Fluids 19, 054105.

AleXakis, A. 2009 Stratified shear flow instabilities at large Richardson numbers. Phys. Fluids 21, 054108.

Balmforth, N. J. \& Mandre, S. 2004 Dynamics of roll waves. J. Fluid Mech. 514, 1-33.

Bretherton, F. P. \& Garrett, C. J. R. 1969 Wavetrains in inhomogeneous moving media. Proc. R. Soc. A 302, 529-554.

BRowand, F. K. \& WinANT, C. D. 1973 Laboratory observations of shear-layer instability in a stratified fluid. Boundary-Layer Meteorol. 5, 67-77.

Carpenter, J. R., Lawrence, G. A. \& Smyth, W. D. 2007 Evolution and mixing of asymmetric Holmboe instabilities. J. Fluid Mech. 582, 103-132.

Caulfield, C. P. \& Peltier, W. R. 2000 Anatomy of the mixing transition in homogenous and stratified free shear layers. J. Fluid Mech. 413, 1-47.

Haigh, S. P. 1995 Non-symmetric Holmboe waves. PhD thesis, University of British Columbia, Vancouver, BC, Canada.

Hazel, P. 1972 Numerical studies of the stability of inviscid parallel shear flows. J. Fluid Mech. 39, $39-61$.

Hogg, A. M. \& IveY, G. N. 2003 The Kelvin-Helmholtz to Holmboe instability transition in stratified exchange flows. J. Fluid Mech. 477, 339-362.

HоLmboe, J. 1962 On the behaviour of symmetric waves in stratified shear layers. Geofys. Pub. 24, $67-112$.

Huang, N. E., Long, S. R. \& Shen, Z. 1996 The mechanism for frequency downshift in nonlinear water wave evolution. Adv. Appl. Mech. 32, 59-117.

HuAng, N. E., Shen, Z. \& Long, S. R. 1999 A new view of nonlinear water waves: the Hilbert spectrum. Annu. Rev. Fluid Mech. 31, 417-457.

KoppeL, D. 1964 On the stability of a thermally stratified fluid under the action of gravity. J. Math. Phys. 5, 963-982.

Lawrence, G. A., Browand, F. K. \& Redekopp, L. G. 1991 The stability of a sheared density interface. Phys. Fluids A 3, 2360-2370.

Longuet-Higgins, M. S. \& CoKelet, E. D. 1978 The deformation of steep surface waves on water. Part II. Growth of normal-mode instabilities. Proc. R. Soc. Lond. A 364, 1-28.

Longuet-Higgins, M. S. \& Dommermuth, D. G. 1997 Crest instabilities of gravity waves. Part 3. Nonlinear development and breaking. J. Fluid Mech. 266, 33-50.

Pawlak, G. \& Armi, L. 1996 Stability and mixing of a two-layer exchange flow. Dyn. Atmos. Oceans 24, 139-151.

Peregrine, D. H. 1976 Interaction of water waves and currents. Adv. Appl. Mech. 16, 9-117.

Pouliquen, O., Chomaz, J. M. \& Huerre, P. 1994 Propagating Holmboe waves at the interface between two immiscible fluids. J. Fluid Mech. 266, 277-302.

Sargent, F. E. \& Jirka, G. H. 1987 Experiments on saline wedge. J. Hydraul. Engng 113, 1307-1324.

Sharples, J., Coates, M. J. \& Sherwood, J. E. 2003 Quantifying turbulent mixing and oxygen fluxes in a Mediterranean-type, microtidal estuary. Ocean Dyn. 53, 126-136. 
Sмyth, W. D. 2006 Secondary circulations in Holmboe waves. Phys. Fluids 37, 064104.

Smyth, W. D., Carpenter, J. R. \& Lawrence, G. A. 2007 Mixing in symmetric Holmboe waves. J. Phys. Oceanogr. 37, 1566-1583.

Smyth, W. D., Klaassen, G. P. \& Peltier, W. R. 1988 Finite amplitude Holmboe waves. Geophys. Astrophys. Fluid Dyn. 43, 181-222.

Smyth, W. D., Moum, J. N. \& Caldwell, D. R. 2001 The efficiency of mixing in turbulent patches: inferences from direct simulations and microstructure observations. J. Phys. Oceanogr. 31, 1969-1992.

Smyth, W. D., Nash, J. D. \& Moum, J. N. 2005 Differential diffusion in breaking Kelvin-Helmholtz billows. J. Phys. Oceanogr. 35, 1004-1022.

Smyth, W. D. \& Peltier, W. R. 1990 Three-dimensional primary instabilities of a stratified, dissipative, parallel flow. Geophys. Astrophys. Fluid Dyn. 52, 249-261.

Smyth, W. D. \& Winters, K. B. 2003 Turbulence and mixing in Holmboe waves. J. Phys. Oceanogr. 33, 694-711.

Tedford, E. W., Carpenter, J. R., Pawlowicz, R., Pieters, R. \& Lawrence, G. A. $2009 a$ Observation and analysis of shear instability in the Fraser River estuary. J. Geophys. Res. 114, C11006. doi:10.1029/2009JC005313.

Tedford, E. W., Pieters, R. \& Lawrence, G. A. 2009b Symmetric Holmboe instabilities in a laboratory exchange flow. J. Fluid Mech. 636, 137-153.

Tulin, M. P. \& WASEDA, T. 1999 Laboratory observations of wave group evolution, including breaking effects. J. Fluid Mech. 378, 197-232.

Wesson, J. C. \& GregG, M. C. 1994 Mixing at the Camarinal Sill in the Strait of Gibraltar. J. Geophys. Res. 99, 9847-9878.

Winters, K. B., MacKinnon, J. A. \& Mills, B. 2004 A spectral model for process studies of rotating, density-stratified flows. J. Atmos. Ocean. Technol. 21, 69-94.

Yonemitsu, N., Swaters, G. E., Rajaratnam, N. \& Lawrence, G. A. 1996 Shear instabilities in arrested salt-wedge flows. Dyn. Atmos. Oceans 24, 173-182.

Yoshida, S., Ohtani, M., Nishida, S. \& Linden, P. F. 1998 Mixing processes in a highly stratified river. In Physical Processes in Lakes and Oceans (ed. J. Imberger). Coastal and Estuarine Studies, vol. 54, pp. 389-400. American Geophysical Union.

Zhu, D. Z. \& Lawrence, G. A. 2001 Holmboe's instability in exchange flows. J. Fluid Mech. 429, 391-409. 